\title{
O índice de produtos de acumulação lipídica como bom preditor de risco cardiovascular em pacientes com doença renal crônica em hemodiálise
}

The lipid accumulation products index as a good predictor of cardiovascular risk in patients with chronic kidney disease in hemodialysis

El índice de productos de acumulación de lípidos como buen predictor del riesgo cardiovascular en pacientes con enfermedad renal crónica en hemodiálisis

\author{
Alexandre Danton Viana Pinheiro \\ ORCID: https://orcid.org/0000-0001-9180-5514 \\ Universidade Estadual do Ceará, Brasil \\ E-mail: alexandredanton@ hotmail.com \\ Francisca Isabelle da Silva e Sousa \\ ORCID: https://orcid.org/0000-0001-6226-3381 \\ Universidade Estadual do Ceará, Brasil \\ E-mail: isabellesilvanutri@gmail.com \\ Dayane Estéfane Barros Albuquerque Linhares \\ ORCID: https://orcid.org/0000-0001-6174-399X \\ Universidade Estadual do Ceará, Brasil \\ E-mail: dayanebarros.linhares@ hotmail.com \\ Ribanna Aparecida Marques Braga \\ ORCID: https://orcid.org/0000-0003-2122-5658 \\ Universidade Estadual do Ceará, Brasil \\ E-mail: ribanna.marques@gmail.com \\ Ruth Silva Galdino \\ ORCID: https://orcid.org/0000-0003-4936-8888 \\ Universidade Estadual do Ceará, Brasil \\ E-mail: ruth.galdino@aluno.uece.br \\ Francisco José Maia Pinto \\ ORCID: https://orcid.org/0000-0003-2976-7857 \\ Universidade Estadual do Ceará, Brasil \\ E-mail: maiapinto@yahoo.com.br \\ Carla Soraya Costa Maia \\ ORCID: https://orcid.org/0000-0003-1535-6686 \\ Universidade Estadual do Ceará, Brasil \\ E-mail: carla.maia@uece.br
}

\begin{abstract}
Resumo
Objetivo: avaliar o poder preditivo do índice Produto de Acumulação Lipídica (Lipid Accumulation Products - LAP) na identificação do risco cardiovascular em pacientes com doença renal em hemodiálise em comparação aos indicadores antropométricos e bioquímicos tradicionais. Metodologia: estudo transversal envolvendo 77 pacientes com Doença Renal Crônica (DRC) em tratamento hemodialíticos. Foram coletados dados de identificação, antropometria e medidas bioquímicas. Indicadores antropométricos, bioquímicos e o que combinam ambos (LAP) foram calculados. Foi realizada curva receiver operating characteristic (ROC) e as áreas sobre a curva foram estimadas a fim de identificar o poder de identificação do Risco Cardiovascular (RCV) e estabelecer pontos de corte dos indicadores. Resultados: A maioria dos indivíduos apresentou risco cardiovascular pela Relação Cintura-Altura (RCA). As medianas de colesterol total e triglicerídeos e a média de LDL-c foram maiores no grupo com RCV avaliado pelo colesterol não-HDL em comparação aos sem RCV ( $p<0,05)$. Escore LAP apresentou diferenças significativas entre os grupos de RCV, sendo a mediana do LAP, 77,54 (45,72-142,88), maior no grupo daqueles com RCV em comparação aos sem RCV, 43,06 (30,98-77,06). LAP (AUC: 0,705; IC95\%: 0,589-0,821; p=0,003) foi o melhor discriminador do RCV, onde o ponto de corte $>92,21$ do LAP, a sensibilidade de $44 \%$ e a especificidade de 92,59\% foram estimados para identificar o RCV. Conclusão: O índice LAP apresentou maior poder na identificação do RCV, quando comparado a medidas antropométricas ou bioquímicas isoladas.

Palavras-chave: Nefropatias; Diálise; Antropometria; Doenças cardiovasculares.
\end{abstract}




\begin{abstract}
Objective: To evaluate the predictive power of the Lipid Accumulation Products (LAP) index in the identification of cardiovascular risk in patients with kidney disease undergoing hemodialysis compared to traditional anthropometric and biochemical indicators. Methods: cross-sectional study involving 77 patients with Chronic Kidney Disease (CKD) undergoing hemodialysis treatment. Identification data, anthropometry and biochemical measurements were collected. Anthropometric, biochemical and combination of both (LAP) indicators were calculated. A receiver operating characteristic (ROC) curve was performed and the areas under the curve were estimated in order to identify the power of identification of Cardiovascular Risk (CVR) and establish cutoff points for the indicators. Results: Most individuals presented cardiovascular risk according to the Waist-Height Ratio (WHR). Medians of total cholesterol and triglycerides and mean LDL-c were higher in the CVR group assessed by non-HDL cholesterol compared to those without CVR $(\mathrm{p}<0.05)$. The LAP score showed significant differences between the CVR groups, with the LAP median being 77.54 (45.72-142.88), higher in the group with CVR compared to those without CVR, 43.06 (30.9877.06). LAP (AUC: 0.705; 95\%CI: 0.589-0.821; $\mathrm{p}=0.003$ ) was the best discriminator of RCV, where LAP cutoff point $>92.21$, sensitivity of $44 \%$ and specificity of $92.59 \%$ were estimated to identify the RCV. Conclusion: The LAP index showed greater power in identifying the CVR when compared to isolated anthropometric or biochemical measurements.
\end{abstract}

Keywords: Kidney disease; Dialysis; Anthropometry; Cardiovascular diseases.

\title{
Resumen
}

Objetivo: Evaluar el poder predictivo del índice de Productos de Acumulación de Lípidos (LAP) en la identificación de riesgo cardiovascular en pacientes con enfermedad renal en hemodiálisis en comparación con indicadores antropométricos y bioquímicos tradicionales. Métodos: estudio transversal en el que participaron 77 pacientes con Enfermedad Renal Crónica (ERC) en tratamiento de hemodiálisis. Se recolectaron datos de identificación, antropometría y medidas bioquímicas. Se calcularon indicadores antropométricos, bioquímicos y combinación de ambos (LAP). Se realizó una curva de característica operativa del receptor (ROC) y se estimaron las áreas bajo la curva para identificar el poder de identificación del Riesgo Cardiovascular (RCV) y establecer puntos de corte para los indicadores. Resultados: La mayoría de los individuos presentó riesgo cardiovascular de acuerdo con el índice cintura-altura (ICC). Las medianas de colesterol total y triglicéridos y la media de LDL-c fueron más altas en el grupo de RCV evaluado por el colesterol no HDL en comparación con aquellos sin RCV (p <0,05). La puntuación LAP mostró diferencias significativas entre los grupos de RCV, siendo la mediana de LAP 77,54 (45,72-142,88), mayor en el grupo con RCV en comparación con aquellos sin RCV, 43,06 (30,98- 77,06). LAP (AUC: 0,705; IC del 95\%: 0,589-0,821; $\mathrm{p}=0,003)$ fue el mejor discriminador de RCV, donde se estimó el punto de corte de LAP> 92,21, la sensibilidad del $44 \%$ y la especificidad del 92,59\% para identificar la RCV. Conclusión: El índice LAP mostró mayor poder en la identificación del RCV en comparación con mediciones antropométricas o bioquímicas aisladas.

Palabras clave: Enfermedades renales; Diálisis; Antropometría; Enfermedades cardiovasculares.

\section{Introdução}

A Doença Renal Crônica (DRC) atinge cerca de 750 milhões de pessoas no mundo e mais de 10 milhões no Brasil Brandão et al. (2021). Na fase terminal da doença, estágio 5 (taxa de filtração glomerular inferior a 15mL/min/1,73m2), é indicada a terapia renal substitutiva, como hemodiálise e diálise peritoneal, ou o transplante renal, visando o aumento na expectativa de vida desses pacientes Brasil (2014).

Ressalta-se que no estágio final da DRC, a combinação de complicações e comorbidades prediz a elevação do risco cardiovascular, de modo que pacientes com DRC possuem maior predisposição ao desenvolvimento de quadros cardíacos mais graves Brandão et al. (2021); Greffin (2017). Neste contexto, enquadra-se a Síndrome Cardiorrenal caracterizada pela presença da disfunção cardíaca e renal Pereira-Rodríguez (2018).

Com intuito de avaliar o RCV, vários índices, antropométricos e bioquímicos, medidos de forma isolada ou em conjunto com outras variáveis, vêm sendo utilizados na triagem da população, tais como, concentrações sanguíneas de triglicerídeos (TG) e lipoproteínas de alta densidade (HDL-c) Almeida et al. (2018) e medidas de circunferência da cintura (CC) e a Relação Cintura-Altura (RCA) Brandão et al. (2021). Destacam-se ainda, os índices emergentes para avaliação do RCV, dentre eles têm-se a Razão Lipídica TG/HDL-c e o Produto de Acumulação Lipídica (Lipid Accumulation Production LAP), o qual se utiliza da associação de marcador antropométrico e de perfil lipídico, na sua formulação. Esses índices correspondem a métodos favoráveis para avaliar e predizer o RCV e cardiometabólico Kahn (2005); Wen et al. (2017). 
Desse modo, diante dos altos índices que associam o risco de desenvolvimento de DCV na população renal, é de grande importância que se estabeleça uma relação entre indicadores antropométricos e perfil lipídico na predição de doença cardiovascular com perspectiva de um diagnóstico precoce e prevenção Brandão (2021).

Tais estratégias que auxiliam na identificação precoce do RCV nessa população são instrumentos de elevada relevância, visto que podem auxiliar na prevenção de determinadas complicações cardiovasculares, as quais representam a principal causa de mortalidade nessa população. O índice LAP pode ser utilizado como ferramentas para subsidiar a atuação na prática clínica dos profissionais da saúde, uma vez que utiliza a combinação de parâmetros de baixo custo e fácil execução. Desse modo, o objetivo desse estudo foi avaliar o poder preditivo do índice LAP na identificação do risco cardiovascular em pacientes com doença renal em hemodiálise em comparação aos indicadores antropométricos e bioquímicos tradicionais.

\section{Metodologia}

\section{Delineamento do estudo, população e aspectos éticos}

Trata-se de um estudo transversal realizado em duas clínicas de referência em uma capital do nordeste brasileiro, no período de junho a agosto de 2015 Estrela (2018). A amostra foi não probabilística por conveniência, constituída por 77 pacientes maiores de 18 anos, com DRC em tratamento dialítico. Foram excluídos pacientes com DRC em outros tipos de tratamento, aqueles diagnosticados com alguma doença infectocontagiosa concomitantes, bem como gestantes, pacientes com nível de consciência alterado e aqueles em que houve incapacidade/dificuldade para realização da técnica antropométrica.

A coleta dos dados foi realizada após assinatura do Termo de Consentimento Livre e Esclarecido. O estudo foi analisado pelo Comitê de Ética e Pesquisa da Secretaria Estadual de Saúde pelo parecer de número 203.906. A pesquisa foi realizada segundo a Resolução do Conselho Nacional de Saúde 466/12 Brasil (2012).

\section{Dados sociodemográficos e estilo de vida}

Foram coletados dados sociodemograficos, como idade, sexo e escolaridade, bem como dados de estilo de vida, histórico de tabagismo e sedentarismo (se praticante ou não de atividade física). Para análise dos dados, o presente estudo classificou a variável escolaridade em baixa e alta, considerando como escolaridade alta, participantes que possuíam o ensino médio completo e/ou ensino superior, e baixa escolaridade, participantes analfabetos ou com ensino médio incompleto.

\section{Dados clínicos}

Em relação aos dados clínicos foram coletadas informações sobre tempo de hemodiálise (HD), presença de Hipertensão Arterial Sistêmica (HAS) e/ou presença de Diabetes Melitos (DM), e os exames bioquímicos de perfil lipídico, tais como: Colesterol total - CT (mg/dL), Triglicerídeos - TGL (mg/dL), Colesterol da Lipoprotéina de Baixa Densidade LDL-c (mg/dL) e Lipoproteína de Alta Densidade Colesterol - HDL-c (mg/dL).

Utilizando o CT e HDL-c foi calculado o Colesterol não HDL-c - Não-HDL-c (mg/dL), segundo a fórmula NãoHDL-c $=[C T(\mathrm{mg} / \mathrm{dl})-\mathrm{HDL}-\mathrm{c}(\mathrm{mg} / \mathrm{dL})]($ PRECOMA, 2019). Para categorização de RCV pelo Não-HDL-c foi considerado pelo estudo os valores maiores ou iguais a $100 \mathrm{mg} / \mathrm{dL}$ como RCV. A utilização do Não-HDL-c para classificação do RCV está respaldada na literatura, em que foram observadas associações do Não-HDL-c com mortalidade em pacientes com DRC em estágios mais avançados Chang et al. (2018); Chiu et al. (2020). Também foram considerados para os exames do perfil lipídico os valores de referência para pacientes de alto risco (com exames laboratoriais realizados em jejum), sendo classificados acima do recomendado valores de CT $\geq 190 \mathrm{mg} / \mathrm{dL}, \mathrm{TGL} \geq 150 \mathrm{mg} / \mathrm{dL}$ e LDL-c $\geq 70 \mathrm{mg} / \mathrm{dL}$, foram considerados valores abaixo do recomendado para HDL-c $\leq 40$ (mg/dL) Sociedade Brasileira de Cardiologia (2017).

O cálculo do índice Razão Triglicerídeo e Lipoproteína de Alta Densidade Colesterol utilizou a seguinte fórmula: 
Razão TGL/HDL-c = [TGL(mg/dL) $\div$ HDL-c (mg/dL)] Millan (2009). O Escore do Produto da Acumulação Lipídica (LAP) foi calculado a partir da CC e TG em jejum, específico por sexo Kahn (2005):

Homens: $(\mathrm{CC}(\mathrm{cm})-65) \times \mathrm{TG}(\mathrm{mmol} / \mathrm{l})$

Mulheres: $(\mathrm{CC}(\mathrm{cm})-58) \times \mathrm{TG}(\mathrm{mmol} / \mathrm{l})$

\section{Medidas antropométricas}

As medidas antropométricas foram realizadas após a sessão de HD. O peso pós sessão de HD foi aferido em balança antropométrica (BALMAK $\square$ ). A altura foi mensurada a partir de estadiômetro acoplado à balança, sendo o paciente posicionado de costa para o equipamento e com a parte posterior do corpo tocando o equipamento, em posição anatômica, descalços e com a cabeça posicionada no plano de Frankfurt (Brasil, 2011). A partir das medidas de peso e altura, foi calculado o IMC $\left(\mathrm{Kg} / \mathrm{m}^{2}\right)\left[\mathrm{IMC}=\right.$ Peso $\left.(\mathrm{Kg}) /(\text { estatura }(\mathrm{m}))^{2}\right]$, sendo considerado excesso de peso valores maiores que $30 \mathrm{Kg} / \mathrm{m}^{2} \mathrm{WHO}$ (1997).

A circunferência da cintura (CC) foi aferida utilizando fita antropométrica inelástica graduada em milímetros, sendo mensurada na região mais estreita do tronco. Para classificação do risco cardiovascular a partir da CC, considerou-se valores maiores ou iguais a $80 \mathrm{~cm}$ para mulheres e maiores ou iguais a $94 \mathrm{~cm}$ para homens BRASIL (2011). Utilizando CC $(\mathrm{cm}) \mathrm{e}$ altura $(\mathrm{cm})$ foi calculada a Relação cintura altura $[\mathrm{RCA}=\mathrm{CC}(\mathrm{cm}) /$ Altura $(\mathrm{cm})]$, classificando-se indivíduos com RCV com valores maiores que 0,5 ABESO (2016).

\section{Análise estatística}

Inicialmente, foram realizadas análises descritivas sendo as variáveis categóricas expressas em frequências e percentuais e as numéricas em medidas de tendência central (médias ou medianas) e de dispersão (desvio padrão ou intervalo interquartil). A normalidade e a homogeneidade dos dados foram verificadas por meio dos testes de Kolmogorov-Smirnov e Levene, respectivamente. As associações entre as variáveis categóricas foram verificadas a partir do teste do qui-quadrado de Pearson, quando as frequências esperadas foram maiores que cinco, e teste exato de Fisher, quando as frequências esperadas foram menores que cinco. Para as comparações de médias entre duas categorias (risco cardiovascular obtido pelo colesterol não-HDL), dependendo da normalidade e homogeneidade, utilizou-se o teste t de Student para amostras independentes ou o teste de Mann-Whitney. As análises foram realizadas utilizando o software estatístico Statistical Package for the Social Sciences (SPSS) versão 22.0, adotando a significância de 5\% ( $\mathrm{p}<0,05)$.

Para identificar o poder discriminatório e propor pontos de corte dos indicadores antropométricos (IMC, CC e RCA), bioquímicos (razão TG/HDL) e o que combina antropométricos e bioquímicos (LAP) para o risco cardiovascular, foi realizado a análise da curva receiver operating characteristic (ROC) e as áreas sobre a curva foram estimadas. Nesta análise, utilizou-se como padrão-ouro o colesterol não-HDL, sendo que os indivíduos foram classificados em com RCV e sem RCV, conforme valores definidos anteriormente. A diferença entre as áreas sobre a curva dos indicadores foi testada segundo teste de Wald. Além disso, os pontos de corte ótimos e os valores de sensibilidade e especificidades dos indicadores foram determinados. Para isso, foi utilizado o software MedCalc versão 19.8 .

\section{Resultados}

O estudo incluiu 77 pacientes, que apresentaram idade mediana de 49 (41-65) anos, sendo composto majoritariamente por mulheres $(55,8 \%)$ e com baixa escolaridade $(84,4 \%)$. Os dados referentes ao estilo de vida mostraram que $58,4 \%$ dos indivíduos não tinham histórico de tabagismo e a maior parte da amostra era sedentária (70,1\%). Em 74\% dos pacientes a hipertensão arterial era presente, mas a maioria $(79,2 \%)$ não tinha diabetes. Não houve associação entre os fatores 
sociodemográficos, de estilo de vida e diagnósticos de comorbidades e a presença de risco cardiovascular (p>0,05) (Tabela 1).

Tabela 1. Caracterização da população do estudo de acordo com ausência ou presença de risco cardiovascular em pacientes com DRC em tratamento dialítico ( $\mathrm{n}=77)$. Nordeste brasileiro, 2015.

\begin{tabular}{|c|c|c|c|c|}
\hline \multirow{2}{*}{ Variáveis } & \multicolumn{4}{|c|}{ Risco Cardiovascular ${ }^{\S}$} \\
\hline & $\begin{array}{l}\text { Total } \\
\text { n }(\%)\end{array}$ & $\begin{array}{c}\text { Sem } \operatorname{risco}(n=27) \\
n(\%)\end{array}$ & $\begin{array}{c}\text { Com } \operatorname{risco}(\mathbf{n}=50) \\
\mathbf{n}(\%)\end{array}$ & $\mathbf{P}$ \\
\hline $\begin{array}{l}\text { Idade (anos), mediana (IIQ) } \\
\text { Sexo }\end{array}$ & $49(41-65)$ & $47(31-71)$ & $50(43,5-61)$ & $0,749^{\#}$ \\
\hline $\begin{array}{l}\text { Masculino } \\
\text { Feminino }\end{array}$ & $\begin{array}{l}34(44,2) \\
43(55,8)\end{array}$ & $\begin{array}{l}16(47,1) \\
11(25,6)\end{array}$ & $\begin{array}{l}18(52,9) \\
32(74,4)\end{array}$ & $0,050^{*}$ \\
\hline \multicolumn{5}{|l|}{ Escolaridade } \\
\hline $\begin{array}{l}\text { Alta } \\
\text { Baixa }\end{array}$ & $\begin{array}{l}12(15,6) \\
65(84,4)\end{array}$ & $\begin{array}{c}6(50,0) \\
21(32,3)\end{array}$ & $\begin{array}{c}6(50,0) \\
44(67,7)\end{array}$ & $0,325^{\mathrm{t}}$ \\
\hline \multicolumn{5}{|l|}{ Histórico de tabagismo } \\
\hline & $\begin{array}{l}32(41,6) \\
45(58,4)\end{array}$ & $\begin{array}{l}15(46,9) \\
12(26,7)\end{array}$ & $\begin{array}{l}17(53,1) \\
33(73,3)\end{array}$ & $0,067 *$ \\
\hline \multicolumn{5}{|l|}{ Prática de atividade física } \\
\hline $\begin{array}{l}\text { Sim } \\
\text { Não }\end{array}$ & $\begin{array}{l}23(29,9) \\
54(70,1)\end{array}$ & $\begin{array}{c}8(34,8) \\
19(35,2)\end{array}$ & $\begin{array}{l}15(65,2) \\
35(64,8)\end{array}$ & $0,973^{*}$ \\
\hline \multicolumn{5}{|l|}{ Presença de HAS } \\
\hline $\begin{array}{l}\text { Sim } \\
\text { Não }\end{array}$ & $\begin{array}{l}57(74,0) \\
20(26,0)\end{array}$ & $\begin{array}{l}21(35,1) \\
6(30,0)\end{array}$ & $\begin{array}{l}36(63,2) \\
14(70,0)\end{array}$ & $0,581^{*}$ \\
\hline \multicolumn{5}{|l|}{ Presença de DM } \\
\hline $\begin{array}{l}\text { Sim } \\
\text { Não }\end{array}$ & $\begin{array}{l}16(20,8) \\
61(79,2)\end{array}$ & $\begin{array}{c}7(43,8) \\
20(32,8)\end{array}$ & $\begin{array}{c}9(56,3) \\
41(67,2)\end{array}$ & \multirow[t]{2}{*}{$0,413 *$} \\
\hline \multicolumn{4}{|l|}{ IMC } & \\
\hline $\begin{array}{l}\text { Sem excesso de peso } \\
\text { Com excesso de peso }\end{array}$ & $\begin{array}{l}66(85,7) \\
11(14,6)\end{array}$ & $\begin{array}{l}25(37,9) \\
2(18,2)\end{array}$ & $\begin{array}{l}41(62,1) \\
9(81,8)\end{array}$ & \multirow[t]{2}{*}{$0,311^{\mathrm{t}}$} \\
\hline \multicolumn{4}{|l|}{$\mathrm{CC}$} & \\
\hline $\begin{array}{l}\text { Sem RCV } \\
\text { Com RCV }\end{array}$ & $\begin{array}{l}42(54,5) \\
35(45,5)\end{array}$ & $\begin{array}{l}19(45,2) \\
8(22,9)\end{array}$ & $\begin{array}{l}23(54,8) \\
27(77,1)\end{array}$ & \multirow[t]{2}{*}{$0,040 *$} \\
\hline \multicolumn{4}{|l|}{ RCA } & \\
\hline $\begin{array}{l}\text { Sem RCV } \\
\text { Com RCV }\end{array}$ & $\begin{array}{l}27(35,1) \\
50(64,9)\end{array}$ & $\begin{array}{l}12(44,4) \\
15(30,0)\end{array}$ & $\begin{array}{l}15(55,6) \\
35(70,0)\end{array}$ & \multirow[t]{2}{*}{$0,205^{*}$} \\
\hline \multicolumn{4}{|l|}{ CT } & \\
\hline $\begin{array}{l}\text { Recomendado } \\
\text { Acima do recomendado }\end{array}$ & $\begin{array}{l}62(80,5) \\
15(19,5)\end{array}$ & $\begin{array}{c}27(43,5) \\
0(0)\end{array}$ & $\begin{array}{l}35(56,5) \\
15(100)\end{array}$ & \multirow[t]{2}{*}{$0,002 *$} \\
\hline \multicolumn{4}{|l|}{ TGL } & \\
\hline $\begin{array}{l}\text { Recomendado } \\
\text { Acima do recomendado }\end{array}$ & $\begin{array}{l}30(39,0) \\
47(61,0)\end{array}$ & $\begin{array}{l}17(56,7) \\
10(21,3)\end{array}$ & $\begin{array}{l}13(43,3) \\
37(78,7)\end{array}$ & $\mathbf{0 , 0 0 2 *}$ \\
\hline \multicolumn{5}{|l|}{ HDL-c } \\
\hline $\begin{array}{l}\text { Recomendado } \\
\text { Abaixo do recomendado }\end{array}$ & $\begin{array}{l}35(45,5) \\
42(54,5)\end{array}$ & $\begin{array}{l}12(34,3) \\
15(35,7)\end{array}$ & $\begin{array}{l}23(65,7) \\
27(64,3)\end{array}$ & \multirow[t]{2}{*}{$0,896^{*}$} \\
\hline \multicolumn{4}{|l|}{ LDL-c } & \\
\hline $\begin{array}{l}\text { Recomendado } \\
\text { Acima do recomendado }\end{array}$ & $\begin{array}{l}35(45,5) \\
42(54,5)\end{array}$ & $\begin{array}{c}24(68,6) \\
3(7,1)\end{array}$ & $\begin{array}{l}11(31,4) \\
39(92,9)\end{array}$ & $<0,001 *$ \\
\hline
\end{tabular}

n, frequência; \%, percentual; IIQ, Intervalo Interquartil; §, Risco cardiovascular obtido pelo colesterol não-HDL-c; HAS, Hipertensão Arterial Sistêmica; DM, Diabetes Mellitus; IMC, Índice de Massa Corporal; CC, Circunferência da Cintura; RCA, Relação Cintura Altura; RCV, Risco Cardiovascular; CT, Colesterol Total; TGL, Triglicerídeo. HDL-c, Colesterol associado à Lipoproteína de Alta Densidade; LDL-c, Colesterol associado à Lipoproteína de Baixa Densidade. p, p valor (p<0,05); * Teste Qui-Quadrado de Pearson; t Teste de Exato de Fisher; \#Teste Mann-Whitney. Fonte: Autores.

Em relação aos indicadores antropométricos, a maioria dos indivíduos não tem excesso de peso segundo IMC, nem risco cardiovascular obtido pela CC, contudo apresenta maior prevalência de risco pela RCA. Quando analisada a categoria de RCV pela CC, observou-se maior prevalência desses indivíduos pertencentes também ao grupo com $R C V$ ( $p<0,05)$. Os valores acima do recomendado de colesterol total, triglicerídeos e LDL-c apresentaram associação significativa com a presença de RCV. Além disso, os valores de mediana das concentrações de colesterol total e LDL-c e as médias de triglicerídeos e dos 
índices LAP e da razão TGL/HDL-c foram significativamente maiores no grupo com RCV em comparação aos sem RCV $(\mathrm{p}<0,05)$ (Tabela 2).

Tabela 2. Comparação entre as médias das variáveis antropométricas, perfil lipídico e índices de RCV, de acordo com ausência ou presença de risco cardiovascular em pacientes com DRC em tratamento dialítico. Nordeste brasileiro, 2015.

\begin{tabular}{|c|c|c|c|c|}
\hline \multirow[b]{2}{*}{ Variáveis } & \multirow[b]{2}{*}{$\begin{array}{c}\text { Total } \\
\text { Média(DP) }\end{array}$} & \multicolumn{2}{|c|}{ Risco Cardiovascular ${ }^{\S}$} & \multirow[b]{2}{*}{$\mathbf{p}$} \\
\hline & & $\begin{array}{c}\text { Sem risco }(n=27) \\
\text { Média(DP) }\end{array}$ & $\begin{array}{c}\text { Com risco }(\mathbf{n}=50) \\
\operatorname{Média}(D P)\end{array}$ & \\
\hline \multicolumn{5}{|l|}{ Antropométricas } \\
\hline Peso $(\mathrm{Kg})$ & $64,03(13,61)$ & $63,16(14,36)$ & $64,50(13,31)$ & $0,690^{t}$ \\
\hline $\mathrm{IMC}_{\left(\mathrm{Kg} / \mathrm{m}^{2}\right)}$ & $25,11(5,21)$ & $24,14(6,05)$ & $25,65(4,68)$ & $0,266^{\mathrm{t}}$ \\
\hline RCA & $0,53(0,07)$ & $0,52(0,08)$ & $0,55(0,08)$ & $0,164^{\mathrm{t}}$ \\
\hline Altura (m), mediana (IIQ) & $1,59(1,53-1,68)$ & $1,65(1,55-1,71)$ & $1,575(1,53-1,65)$ & $0,051^{\#}$ \\
\hline $\mathrm{CC}_{(\mathrm{cm})}$, mediana $(\mathrm{IIQ})$ & $86(76-95)$ & $84(76-92)$ & $86,75(76-96,63)$ & $0,390^{\#}$ \\
\hline \multicolumn{5}{|l|}{ Perfil lipídico } \\
\hline $\mathrm{CT}_{(\mathrm{mg} / \mathrm{dL})}$, mediana (IIQ) & $148(125-183)$ & $118(104-128)$ & $172(148-204,5)$ & $<\mathbf{0 , 0 0 1}{ }^{\#}$ \\
\hline $\mathrm{LDL}_{(\mathrm{mg} / \mathrm{dL})}$, mediana (IIQ) & $72,70(52,10-99,50)$ & $48(37,4-67)$ & $88,85(72,37-113,3)$ & $<0,001^{\#}$ \\
\hline TGL & $195,72(118,54)$ & $142,06(38,62)$ & $224,71(136,24)$ & $0,003^{t}$ \\
\hline HDL-c & $38,97(12,11)$ & $38,15(11,69)$ & $39,42(12,43)$ & $0,658^{\mathrm{t}}$ \\
\hline \multicolumn{5}{|l|}{ Índices de RCV } \\
\hline Razão TG/HDL, mediana (IIQ) & $4,38(3,25-6,68)$ & $3,84(2,66-5,29)$ & $4,98(3,43-7,89)$ & $\mathbf{0 , 0 2 0} 0^{\#}$ \\
\hline LAP, mediana (IIQ) & $62,46(37,20-110,52)$ & $43,06(30,98-77,06)$ & $77,54(45,72-142,88)$ & $\mathbf{0 , 0 0 3}{ }^{\#}$ \\
\hline
\end{tabular}

DP, Desvio Padrão; IIQ, Intervalo Interquartil; §, Risco cardiovascular obtido pelo colesterol não-HDL-c; IMC, Índice de Massa Corporal; CC, Circunferência da Cintura; RCA, Relação Cintura Altura; RCV, Risco Cardiovascular; CT, Colesterol Total; TGL, Triglicerídeo. HDL-c, Colesterol associado à Lipoproteína de Alta Densidade; LDL-c, Colesterol associado à Lipoproteína de Baixa Densidade; Razão TGL/HDL, Razão Triglicerídeo e Colesterol associado à Lipoproteína de Alta Densidade; LAP, Produção de Acumulação Lipídica. p, p valor (p<0,05); \# Teste Mann-Whitney; Teste de t-Student para amostras independentes. Fonte: Autores.

$\mathrm{Na}$ análise da curva ROC, observou-se que os indicadores antropométricos (IMC, CC e RCA) não apresentaram poder discriminatório significativo para identificar o RCV ( $<<0.05)$. Contudo, o indicador bioquímico razão TGL/HDL-c e o índice LAP apresentaram bom desempenho, sendo o LAP o melhor discriminador do RCV, por apresentar maior área sobre a curva ROC, (Tabela 3) com um ponto de corte estimado para identificar esse risco de $92,59 \%$, sensibilidade de $44 \%$ e especificidade de $92,59 \%$ (Tabela 4$)$.

Tabela 3. Comparação das áreas sobre a curva com respectivos intervalos de confiança dos índices antropométricos e bioquímicos para identificar o risco cardiovascular obtido pelo Colesterol não HDL-c.

\begin{tabular}{lccc}
\hline \multicolumn{1}{c}{ Índices } & AUC $($ IC 95\%) & Erro Padrão & p \\
\hline IMC & $0,608(0,469-0,746)$ & 0,070 & 0,120 \\
CC & $0,560(0,427-0,692)$ & 0,067 & 0,390 \\
RCA & $0,610(0,474-0,747)$ & 0,069 & 0,112 \\
Razão TGL/HDL-c & $0,661(0,537-0,786)$ & 0,063 & $\mathbf{0 , 0 2 0}$ \\
LAP & $0,705(0,589-0,821)$ & 0,059 & $\mathbf{0 , 0 0 3}$ \\
\hline
\end{tabular}

AUC, Área sobre a Curva; IC 95\%, Intervalo de Confiança de 95\%; IMC, Índice de Massa Corporal; CC, Circunferência da Cintura; RCA, Relação Cintura Altura; Razão TGL/HDL-c, Razão Triglicerídeo e Colesterol associado à Lipoproteína de Alta Densidade; LAP, Produção de Acumulação Lipídica; p, p valor $(p<0,05)$. Fonte: Autores. 
Tabela 4. Pontos de corte, sensibilidades e especificidades dos índices LAP e Razão TG/HDL para identificação de RCV.

\begin{tabular}{lccc}
\hline \multicolumn{1}{c}{ Índices } & Ponto de corte & Sensibilidade $(\boldsymbol{\%})$ & Especificidade $(\boldsymbol{\%})$ \\
\hline LAP & $>92,21$ & 44,00 & 92,59 \\
Razão TG/HDL-c & $>6,08$ & 38,00 & 88,89 \\
\hline
\end{tabular}

Razão TGL/HDL-c, Razão Triglicerídeo e Colesterol associado à Lipoproteína de Alta Densidade; LAP, Produção de Acumulação Lipídica. Fonte: Autores.

\section{Discussão}

O presente estudo mostrou melhor desempenho do LAP na predição de RCV, em pacientes com DRC em tratamento dialítico quando comparado a medidas antropométricas isoladas (CC) e suas relações (IMC e RCA), bem como a Razão TGL/HDL-c. De forma semelhante, Biyik e Guney (2019) observaram que o mesmo índice apresentou melhor desempenho para identificar a presença de síndrome metabólica em indivíduos de ambos os sexos e com doença renal, também em comparação com análise de variáveis isoladas, como IMC e CC, com AUC de 0,908 para LAP, sensibilidade de 81,8\%, especificidade $86,2 \%$ e pontos de corte ideais de 33,5 em mulheres e 36,6 em homens.

Outros pontos de corte existentes na literatura são relatados, como o de Motamed e colaboradores (2016), estabelecendo o ponto de corte para LAP de 49,71 para mulheres, com sensibilidade de $85,2 \%$ e especificidade $82,3 \%$ e 39,89 para homens, com sensibilidade de $86 \%$ e especificidade $79,6 \%$, em população iraniana, onde mostrou desempenho significativamente maior do LAP (AUC: 0,904) em encontrar síndrome metabólica comparados a medidas individuais de obesidade, como triglicerídeos. Guo e outros autores (2016) determinaram pontos de corte ideais para o LAP, sendo 34,7 (AUC: 0,853 , sensibilidade 73,93\% e especificidade 83,15\%) e para homens e 27,3 (AUC: 0,817, sensibilidade 78,88\% e especificidade $69,71 \%$ ) para mulheres, a fim de identificar a presença de Síndrome Metabólica em população rural e de baixa renda da China.

Zhang e colaboradores (2016), divergindo dos achados do presente estudo, observaram que o LAP e a RCA detinham poder preditor similar na detecção de critérios para SM em população asiática, considerando valor de AUC 0,853 e encontraram pontos de corte de 38,3 (sensibilidade $76,6 \%$ e especificidade 78,9\%) e 28,2 (sensibilidade 78,6\% e especificidade 77,5\%) para população de Cazaques e Uigures, respectivamente. Major e colaboradores (2018) demonstraram que as medições de lipídios séricos, colesterol total e LDL-c, não obtiveram uma relação clara para detecção de RCV na população com doença renal, frisando a importância de envolver mais variáveis a fim de predizer tal possível risco.

Além disso, vale ressaltar que a avaliação do estado nutricional, mesmo quando realizada após a sessão de HD, e aumento do RCV podem ser afetadas pelo ganho de peso interdialítico e pela hiper-hidratação, que em muitos casos, pode estar associada à perda de massa muscular e ao aumento dos níveis pressóricos. (Davies \& Davenport, 2014).

O índice LAP considera a circunferência da cintura, parâmetro de avaliação da obesidade central (ABESO, 2016), e o marcador bioquímico, triglicerídeo, que está associado à presença de gordura ectópica, que por sua vez, está relacionada à dislipidemia, diabetes e doenças cardiovasculares. (Zou et al. 2020). A obesidade abdominal ou central está relacionada ao comprometimento do funcionamento renal, induzindo alterações estruturais que promovem a hipertensão glomerular, aumento da Taxa de Filtração Glomerular (TFG) e a reabsorção tubular de sódio. (D’Agati et al. 2016; Bagasrawala et al. 2019).

Zhang et al. (2017) avaliaram a capacidade de parâmetros de obesidade na predição da progressão da disfunção renal e observaram que o LAP foi associado fortemente a TFG e redução da função renal. Em outro estudo foi observado a associação dos níveis elevados de LAP e o aumento da albuminúria na população pré-diabética Liu et al. (2021).

Lin et al. (2020) observaram que, em pacientes em HD crônica, a obesidade central esteve associada a fatores de risco cardiometabólico. Entretanto, não houve associação da obesidade central ao aumento da incidência de eventos 
cardiovasculares, como acidente vascular cerebral, infarto agudo do miocárdio. A disfunção renal resulta no aumento da síntese tubular de ácidos graxos provocando acúmulo de lipídios. A dislipidemia e as alterações no metabolismo de lipídios são prevalentes em pacientes com DRC, sugerindo que a lipotoxicidade seja o mecanismo pelo qual, vários órgãos, como coração, fígado e tecido muscular, sejam afetados durante a progressão da DRC. (Nishi et al., 2019).

Em pacientes com obesidade e/ou DRC a interrupção da homeostasia, pela liberação de marcadores inflamatórios, resulta na inflamação sistêmica, que ocasiona a disbiose e o aumento da permeabilidade intestinal, amplificando as respostas inflamatórias. (Câmara et al., 2017). As alterações da microbiota intestinal ocasionam disfunções cardiovasculares e renais por estimular um estado inflamatório sistêmico, uma vez que são liberadas citocinas pró-inflamatórios e toxinas urêmicas, dentre outros. (Sumida \& Kovesdy. 2019). Essa amplificação do estado inflamatório resulta em alterações vasculares que aumentam o RCV, como a rigidez vascular, aterosclerose e hipertensão. (Zanoli et al. 2019; Bartekova et al., 2019).

Em nosso trabalho identificamos que os valores de colesterol total, LDL e TGL foram maiores no grupo com RCV em comparação aos sem RCV. Em sua população com DRC, Mondal e colaboradores (2021) encontraram alteração lipídica, principalmente TGL onde, através desse marcador, o desenvolvimento do RCV esteve presente em $89 \%$ da população estudada. $\mathrm{O}$ uso de fármacos hipolipemiantes com foco em redução de colesterol foi eficaz em reduzir o RCV e Doenças Arterial Coronariana em DRC, conforme achado observado por Baigent e colaboradores (2011). O adequado gerenciamento do risco cardiovascular, evidenciado por alterações no perfil lipídico, requer acompanhamento em equipes multiprofissionais, principalmente através da utilização de terapias hipolipemiantes KDIGO (2013).

O estudo não contou com todos os pacientes da clínica por necessitar de exames em jejum, o que inviabilizou a inclusão dos participantes do período da tarde e também não realizou análise de citocinas ou de microbiota que poderiam esclarecer melhor o comportamento dos pacientes em relação ao RCV. No entanto, o estudo foi conduzido com rigor metodológico por pesquisadores treinados que reforçam a qualidade dos achados. Assim, ressaltamos a importância da análise de utilização do índice LAP, principalmente por considerar medições antropométricas associadas a bioquímicas, uma vez considerado a existência de poucos estudos envolvendo a população com DRC na literatura. Ainda, ressalta-se a utilização de um parâmetro de baixo custo, que pode ser utilizado na prática clínica, para triagem de pacientes portadores de DRC em tratamento dialítico.

\section{Conclusão}

Portanto, o índice LAP, que combina parâmetros antropométricos e bioquímicos, apresentou maior poder preditivo na identificação do RCV em pacientes com doença renal em hemodiálise em comparação aos indicadores antropométricos e bioquímicos tradicionais. Como sugestões para futuros trabalhos, podemos ressaltar a análise de parâmetros bioquímicos de função renal, bem como marcadores clínicos que poderiam interferir no RCV, visto a possibilidade de intervenção precoce no desenvolvimento de RCV em pacientes com DRC.

\section{Referências}

Associação Brasileira para o Estudo da Obesidade e da Síndrome Metabólica - ABESO (2016). Diretrizes brasileiras de obesidade. Associação Brasileira para o estudo da Obesidade e da Síndrome Metabólica. (4a ed.), 4: 1-188.

C Almeida, A. F., Sena, M. H. L. G., Santana, G. T., Barbosa, R. L., Gobatto, A. L. N., Conceição, M. E. P., et al (2017). Razão TG/HDL-c, indicadores antropométricos e bioquímicos de risco cardiovascular no renal crônico em tratamento conservador. Nutricion Clinica y Dietetica Hospitalaria, 37(4): 10-16.

Bagasrawala, S. I., Sheth, H., Shah, H., Ansari, R., \& Lakdawala, M (2019). Metabolic Syndrome Rather than Obesity Alone Is More Significant for Kidney Disease. Obes Surg, 29(11):3478-3483. 10.1007/s11695-019-04011-2.

Baigent, C. et al (2011). "The effects of lowering LDL cholesterol with simvastatin plus ezetimibe in patients with chronic kidney disease (Study of Heart and Renal Protection): a randomised placebo-controlled trial.” Lancet, 377,9784: 2181-92. 10.1016/S0140-6736(11)60739-3. 
Bartekova, M., Radosinska, J., Jelemensky, M., \& Dhalla, N. S (2018). Role of cytokines and inflammation in heart function during health and disease. Heart Fail Rev, 23(5):733-758. 10.1007/s10741-018-9716-x.

Biyik, Z., \& Guney, I (2019). Lipid accumulation product and visceral adiposity index: two new indices to predict metabolic syndrome in chronic kidney disease. Eur Rev Med Pharmacol Sci, 23(5):2167-2173. 10.26355/eurrev_201903_17262.

Brandão, H. F. C., Saraiva, M. B. M., Sousa, B. S., Almeida, S. S., Souza, E. D. S., Melo, H. C. M., et al (2021). Estado nutricional e sua associação com risco cardiovascular no paciente em tratamento hemodialítico. Brazilian Journal Of Development, 7(2): 11712-11728.

Brasil - Ministério da Saúde (2011). Orientações para a coleta e análise de dados antropométricos em serviços de saúde: Norma técnica do Sistema de Vigilância Alimentar e Nutricional - SISVAN. Ministério da Saúde, Secretaria de Atenção à Saúde, Departamento de Atenção Básica, Ministério da Saúde, $76 \mathrm{p}$.

Câmara, N. O., Iseki, K., Kramer, H., Liu, Z. H., \& Sharma, K (2017). Kidney disease and obesity: epidemiology, mechanisms and treatment. Nat Rev Nephrol, 13(3):181-190. 10.1038/nrneph.2016.191. Epub 2017.

Chang, T. I., Streja, E., Ko, G. J., Naderi, N., Rhee, C. M., Kovesdy, C. P. et al (2018). Inverse Association Between Serum Non-High-Density Lipoprotein Cholesterol Levels and Mortality in Patients Undergoing Incident Hemodialysis. J Am Heart Assoc, 7:e009096. 10.1161/JAHA.118.009096.

Chiu, H., Wu, P. Y., Huang, J. C., Tu, H. P., Lin, M. Y., Chen, S. C. C., \& Chang, J. M (2020). There is a U shaped association betweem non high densitu lipoprotein cholesterel with overall and cardiovascular mortality in chronic kidney disease stage 3-5. Scientifc Reports, 10(1):1-11.

Conselho Nacional de Saúde (BRASIL). Resolução $\mathrm{n}^{\circ} \quad 466$, de 12 de dezembro de $2012 . \quad$ Brasília, 2012. http://www.conselho.saude.gov.br/resolucoes/reso_12.htm.

D'Agati, V. D., Chagnac, A., de Vries, A. P., Levi, M., Porrini, E., Herman-Edelstein, M., \& Praga, M (2016). Obesity-related glomerulopathy: clinical and pathologic characteristics and pathogenesis. Nat Rev Nephrol, 12(8):453-71. 10.1038/nrneph.2016.75.

Davies, S. J., \& Davenport, A (2014). The role of bioimpedance and biomarkers in helping to aid clinical decision-making of volume assessments in dialysis patients. Kidney Int, 86(3):489-96. 10.1038/ki.2014.207.

Estrela, C. Metodologia científica: ciência, ensino, pesquisa Artes Médicas, 738p.

Greffin, S., André, M. B., Matos, J. P. S., Kang, H. C., Jorge, A. J. L., Rosa, M. L. G., et al (2017). Chronic kidney disease and metabolic syndrome as risk factors for cardiovascular disease in a primary care program. J Bras Nefrologia, 39(3): 246-252.

Guo, S. X., Zhang, X. H., Zhang, J. Y., He, J., Yan, Y. Z., Ma, J. L., Ma, R. L., Guo, H., Mu, L. T., Li, S. G., Niu, Q., Rui, D. S., Zhang, M., Liu, J. M., Wang, K., Xu, S. Z., Gao, X., \& Ding, Y. S (2016). Visceral Adiposity and Anthropometric Indicators as Screening Tools of Metabolic Syndrome among Low Income Rural Adults in Xinjiang. Sci Rep, 26;6:36091.

Kahn, H. S (2005). The lipid accumulation product performs better tha the body mass index for recognizing cardiovascular risk: a population-based comparison. BMC Cardiovasc Disord. 5(26), doi:10.1186/1471-2261-5-26.

KDIGO (2014). Kidney Disease: Improving Global Outcomes (KDIGO) Lipid Working Group. KDIGO clinical practice guideline for lipid management in chronic kidney disease. Kidney Int. Suppl, 3, 263-305.

Lin, T. Y., Hung, S. C., Lim, P. S (2019). Central obesity and incident atherosclerotic cardiovascular disease events in hemodialysis patients. Nutr Metab Cardiovasc Dis, 9;30(3):500-507. 10.1016/j.numecd.2019.11.004.

Liu, Y., Wang, Y., Wang, J., Chen, K., Jin, L., Wang, W., Gao, Z., Tang, X., Yan, L., Wan, Q., Luo, Z., Qin, G., Chen, L., \& Um, Y (2021). Lipid Accumulation Product is Associated with Urinary Albumin-creatinine Ratio in Chinese Prediabitic Population: A Report from the REACTION Study. Diabetes Metab Syndr Obes, 28;14:2415-2425. 10.2147/DMSO.S310751.

Major, R. W., Cheng, M. R. I., Grant, R. A., Shantikumar, S., Xu, G., Oozeerally, I., Brunskill, N. J., \& Gray, L. J (2018). Cardiovascular disease risk factors in chronic kidney disease: A systematic review and meta-analysis. PLoS One, 21;13(3):e0192895. 10.1371/journal.pone.0192895.

Millan, J., Pinto, X., Munoz, A., Zuniga, M., Rubies-Prat, J., Pallardo, L. F., et al (2009). Lipoprotein ratios: Physiological significance and clinical usefulness in cardiovascular prevention. Vasc Health Risk Manag, 5:757-65.

Ministério da Saúde. Secretaria de Atenção à Saúde. Departamento de Atenção Especializada e Temática. Diretrizes Clínicas para o Cuidado ao paciente com Doença Renal Crônica - DRC no Sistema Único de Saúde. Brasília (Brasil) 2014; 37.

Mondal, E. et al (2021). "The Pattern of Lipid Profile in Patients with Chronic Kidney Disease”. Mymensingh medical journal MMJ, 30(1): 48-55.

Motamed, N., Razmjou, S., Hemmasi, G., Maadi, M., \& Zamani, F (2015). Lipid accumulation product and metabolic syndrome: a population-based study in northern Iran, Amol. J Endocrinol Invest, 39(4):375-82. 10.1007/s40618-015-0369-5.

Nishi, H., Higashihara, T., \& Inagi, R (2019). Lipotoxicity in Kidney, Heart, and Skeletal Muscle Dysfunction. Nutrients, 20;11(7):1664. $10.3390 /$ nu11071664.

Pereira-Rodríguez, J. E., Boada-Morales, L., Niño-Serrato, D. R., Caballero-Chavarro, M., Ricón-Gonzalez, G., Jaimes-Martín, T., et al (2017). Síndrome cardiorrenal. Rev Colomb Cardio, 24(6): 602-613.

Précoma, D. B., Oliveira, G. M. M., Simão, A. F., Dutra, O. P., Coelho, O. R., Izar, M. C. O., et al (2019). Updated Cardiovascular Prevention Guideline of the Brazilian Society of Cardiology - 2019. Arq Bras Cardiol, 113(4): 787-891. 
Research, Society and Development, v. 11, n. 2, e10611225401, 2022

(CC BY 4.0) | ISSN 2525-3409 | DOI: http://dx.doi.org/10.33448/rsd-v11i2.25401

Sociedade Brasileira de Cardiologia (2017). Atualização da Diretriz brasileira de dislipidemia e prevenção da aterosclerose. Arquivos Brasileiros de Cardiologia, 109(2):1-92

Sumida, K., \& Kovesdy, C. P (2019). The gut-kidney-heart axis in chronic kidney disease. Physiol Int, 1;106(3):195-206. 10.1556/2060.106.2019.19.

Wen, J., Chen, Y., Huang, Y., Lu, Y., Liu, X., Zhou, H., et al (2017). Association of the TG/HDL-C and Non-HDL-C/HDL-C Ratios with Chronic Kidney Disease in an Adult Chinese Population. Kidney And Blood Pressure Research, 42(6):1141-1154.

World Health Organization (1997). Obesity. Preventing and managing the global epidemic. Geneva: report of a WHO consultation on obesity.

Zanoli, L., Lentini, P., Briet, M., Castellino, P., House, A. A., London, G. M., Malatino, L., McCullough, P. A., Mikhailidis, D. P., \& Boutouyrie, P (2019). Arterial Stiffness in the Heart Disease of CKD. J Am Soc Nephrol, 30(6):918-928. 10.1681/ASN.2019020117.

Zhang, K., Li, Q., Chen, Y., Wang, N., \& Lu, Y (2017). Visceral adiposity and renal function: an observational study from SPECT-China. Lipids Health Dis, 27;16(1):205. 10.1186/s12944-017-0597-0.

Zhang, X. H., Zhang, M., He, J., Yan, Y. Z., Ma, J. L., Wang, K., Ma, R. L., Guo, H., Um, L. T., Ding, Y. S., Zhang, J. Y., Liu, J. M., Li, S. G., Niu, Q., Rui, D. S., \& Guo, S. X (2016). Comparison of Anthropometric and Atherogenic Indices as Screening Tools of Metabolic Syndrome in the Kazakh Adult Population in Xinjiang. Int J Environ Res Public Health, 16;13(4):428. 10.3390/ijerph13040428.

Zou, Y., Sheng, G., Yu, M., \& Xie, G (2020). The association between triglycerides and ectopic fat obesity: An inverted U-shaped curve. PLoS One, 30;15(11):e0243068. 10.1371/journal.pone.0243068. 